\title{
Phenotypic and molecular identification of carbapenemase-producing Enterobacteriaceae - challenges in diagnosis and treatment
}

\author{
Annamária Főldes ${ }^{1 *}$, Doina-Veronica Bilca ${ }^{2}$, Edit Székely ${ }^{2,3}$ \\ 1. Department of Microbiology, Laboratory of Medical Analysis, ’Dr. Constantin Opriş’ County \\ Emergency Hospital Baia Mare, Romania \\ 2. Department of Microbiology, Central Clinical Laboratory, Tîrgu-Mureş County Emergency Hos- \\ pital Clinics, Romania \\ 3. Department of Microbiology, University of Medicine and Pharmacy Tîrgu-Mureş, Romania
}

\begin{abstract}
Introduction: Infections due to carbapenemase-producing carbapenem-resistant Enterobacteriaceae (CPCRE) are an emerging global public health threat. The purpose of this study was to investigate phenotypic and genotypic features of CP-CRE strains isolated from hospitalized patients.

Material and methods: Between $1^{\text {st }}$ of January - $1^{\text {st }}$ of July 2017, in the Department of Microbiology, "Dr. Constantin Opriş" County Emergency Hospital Baia Mare, Romania, 1110 strains of Enterobacteriaceae were isolated from bronchial secretions, urine, wounds and blood cultures. Bacterial identification and antimicrobial susceptibility tests were performed by conventional methods, Vitek 2 Compact and M.I.C.E. strips. We analysed all Enterobacteriaceae strains non-susceptible to carbapenems according to CLSI 2017 criteria. The modified Hodge test (MHT), the modified carbapenem inactivation method ( $m C I M)$ and the combination disks test (KPC, MBL, OXA-48 Confirm kit, Rosco Diagnostica) were used for phenotypic confirmation, whereas a multiplex PCR assay for genes blaKPC, blaNDM and blaOXA-48 was used for genetic confirmation.

Results: 19 non-duplicate strains isolated from 16 patients were phenotypically identified as CP-CRE: Klebsiella pneumoniae $(n=14)$, Escherichia coli $(n=2)$, Providencia stuartii $(n=2)$ and Serratia marcescens $(n=1)$. Most strains were isolated from bronchial secretions $(n=9)$. The carbapenem-hydrolizing enzymes were identified by the combination disks test as: KPC $(n=9), O X A-48$-like $(n=5)$ and MBL $(n=5)$. Molecular confirmation was performed in 18 phenotypically positive isolates with $100 \%$ concordant results with $m$ CIM and combination disks test. Discrepant results were noticed with the MHT in case of 4 NDM-producers confirmed by PCR. All CP-CRE strains were resistant to all tested cephems. Three out of $9 \mathrm{~K}$. pneumoniae strains tested against colistin were found resistant.

Conclusions: The most common carbapenemase detected was KPC. Therapeutic options were limited in all positive cases. Rapid and reliable detection of CP-CRE is critical for preventing the spread of these pathogens.
\end{abstract}

Keywords: carbapenemases, Enterobacteriaceae, carbapenem resistance

Received: $18^{\text {th }}$ February 2018; Accepted: 13 th April 2018; Published: $17^{\text {th }}$ April 2018

*Corresponding author: Annamária Főldes, ”Dr. Constantin Opriş" County Emergency Hospital Baia Mare, Romania. E-mail: anafoldes@yahoo.com 


\section{Introduction}

Gram-negative bacilli belonging to the Enterobacteriaceae family are members of the normal colonic microbiota and these agents can be involved in virtually any type of human infections including urinary tract, bloodstream, intraabdominal, musculoskeletal, device-associated infections, pneumonia, meningitis, in both community and hospital settings $(1,2)$.

Carbapenems are considered the most reliably active treatment option against severe infections caused by multidrug-resistant and extensively drug-resistant Gram-negative bacilli (2-4). In Enterobacteriaceae, carbapenem resistance develops via two main mechanisms: (i) acquisition of carbapenemase genes that encode enzymes capable of degrading carbapenems, or (ii) overexpression of $\beta$-lactamases that possess very weak affinity for carbapenems coupled with modifications in permeability of porins and/or expression of efflux pumps (1).

The worldwide emergence and spread of CP-CRE strains are an important growing public health concern due to very limited treatment options of infections caused by them (2-5). Carbapenemase genes are usually encoded on large transferable plasmids and are associated with various mobile genetic structures (insertion sequences, integrons, transposons), further enhancing their spread (1). CP-CRE isolates are often resistant to all $\beta$-lactam drugs and frequently carry additional mechanisms conferring resistance to other antimicrobial classes (2).

The most clinically important carbapenemases encountered in Enterobacteriaceae are categorized according to the Ambler classification system as follows: (i) class A serine $\beta$-lactamases (Klebsiella pneumoniae carbapenemases KPC), (ii) class B metallo- $\beta$-lactamases (New Delhi metallo- $\beta$-lactamase NDM, Verona integron-encoded metallo- $\beta$-lactamase VIM, Imi- penemase IMP), and (iii) class $\mathrm{D}$ oxacillinases (OXA-48-like and its variants) $(1,2,6)$.

KPC enzymes emerged in North Carolina USA in 1996 and now are found worldwide, the principal reservoirs of $b l a_{\mathrm{NDM}}$ genes are the Indian subcontinent, the Balkan states and the Middle East and the OXA-48 type enzymes circulating among Mediterranean countries and being progressively disseminated to other geographical areas $(1,5)$.

Recently, the World Health Organization (WHO) included CRE among pathogens with critical priority for research and development of new antibiotics (7). Therefore, highly sensitive and specific methods for CP-CRE detection in clinical laboratory settings are critical $(2,4,8)$.

A limited number of published studies from our country about the geographical distribution, spread and type of carbapenemases are available (9-13).

The aim of this study was to investigate phenotypic and genotypic characteristics of CPCRE strains isolated from patients admitted to our hospital. In 2015, we diagnosed phenotypically our first $3 \mathrm{CP}-\mathrm{CRE}$ strains. In this paper, we report our first CP-CRE isolates that were molecularly confirmed.

\section{Material and methods}

\section{Study design}

We conducted a retrospective study between $1^{\text {st }}$ of January $-1^{\text {st }}$ of July 2017 in the Department of Microbiology of "Dr. Constantin Opriș" County Emergency Hospital Baia Mare, Romania, which is a 920-bedded non-teaching hospital with surgical, orthopaedic, medical, haemato-oncological, paediatric and intensive care services. During the study period 1110 strains of Enterobacteriaceae were isolated from bronchial secretions, urine, wounds and blood cultures. 


\section{Bacterial strains and antimicrobial suscep- tibility testing}

Bacterial identification to the species level was performed by conventional methods including Vitek 2 Compact (bioMérieux, France) and API 20E (bioMérieux, France). Antibiotic susceptibility tests were done using disk-diffusion method, Vitek 2 Compact and M.I.C.E. strips with meropenem (Oxoid, UK). The minimal inhibitory concentration (MIC) to tigecycline was determined using Vitek AST-XN05 cards. The results were interpreted according to the Clinical and Laboratory Standards Institute (CLSI) 2017 criteria (14).

\section{Phenotypic detection of resistance mecha- nisms}

In all Enterobacteriaceae strains non-susceptible to carbapenems, we performed the modified Hodge test (MHT) and the modified carbapenem inactivation method (mCIM) (14). The combination disks test (KPC, MBL, OXA48 Confirm kit, Rosco Diagnostica) was used for phenotypic confirmation of carbapenem-hydrolizing enzymes according to the manufacturer's instructions. Extended-spectrum $\beta$-lactamases (ESBLs) production was signaled by the automated system Vitek and was also evaluated by CLSI screening and confirmatory test methods (14).

\section{Molecular analysis of carbapenemase genes}

Isolates stored at $-70^{\circ} \mathrm{C}$ were subcultured and DNA was extracted from bacteria grown in $24 \mathrm{~h}$ culture on solid medium. Multiplex PCR assays for carbapenemase encoding genes ( $b l a_{\mathrm{KPC}}$, $b l a_{\mathrm{NDM}}$ and $\left.b l a_{\mathrm{OXA}-48-\mathrm{like}}\right)$ was carried out as described elsewhere (9).

\section{Results}

\section{CP-CRE strains}

During the study period, we identified 43 carbapenem non-susceptible Enterobacteriaceae isolates from which only 19 non-duplicate strains recovered from 16 patients were phenotypically confirmed as CP-CRE as follows: Klebsiella pneumoniae ( $\mathrm{n}=14 ; 73.68 \%)$, Escherichia coli $(\mathrm{n}=2 ; 10.52 \%)$, Providencia stuartii $(\mathrm{n}=2 ; 10.52 \%)$ and Serratia marcescens $(\mathrm{n}=1$; $5.26 \%$ ). Fourteen strains out of a total of 155 K. pneumoniae isolates were CP-CRE (9.03\%). Of the remaining 24 carbapenem non-susceptible Enterobacteriaceae strains, more than half ( $\mathrm{n}=16,66.66 \%)$ were Enterobacter spp.

The CP-CRE strains were collected from bronchial secretions $(n=9 ; 47.36 \%)$, urine $(n=4$; $21.05 \%)$, wounds $(\mathrm{n}=4 ; 21.05 \%)$ and blood cultures $(n=2 ; 10.52 \%)$. These strains were isolated from patients hospitalized in the intensive care unit (ICU) $(\mathrm{n}=12 ; 63.15 \%)$, medical wards $(\mathrm{n}=5$, $26.31 \%)$ and surgical units $(n=2,10.52 \%)$.

\section{Phenotypic and molecular CP-CRE detec- tion methods}

MHT was positive in 15 of 19 CP-CRE strains with both meropenem and ertapenem disks (Figure 1A). The mCIM was positive in all 19 CP-CRE strains and no false-positive results were noticed (Figure 1B).

The carbapenem-hydrolizing enzymes were identified by the combination disks test (Rosco Diagnostica) as follows: KPC ( $\mathrm{n}=9,47.36 \%)$, OXA-48-like $(n=5,26.31 \%)$ and MBL $(n=5$, 26.31\%). All five OXA-48-like isolates coproduced ESBL.

Carbapenemase-encoding genes were confirmed by multiplex PCR analysis in 18 available isolates with $100 \%$ agreement with the mCIM and combination disks test. The five MBL strains harbored $b l a_{\mathrm{NDM}}$ genes. No multiple carbapenemase genes in the same strain were identified. 

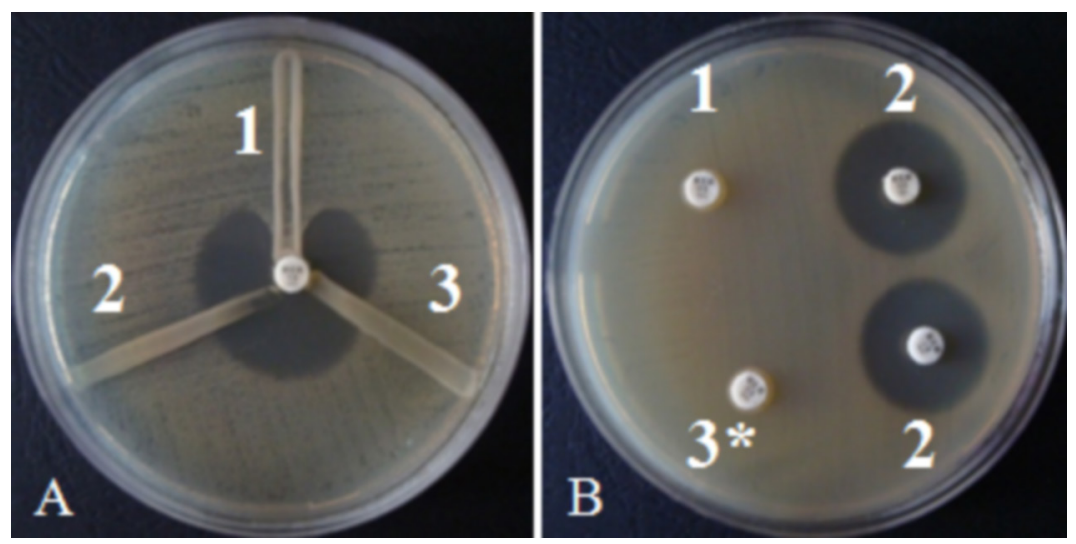

Figure 1. Phenotypic carbapenemase detection by MHT (1A) and MCIM (1B)

Legend: 1: Positive control: $K$. pneumoniae ATCC BAA 1705; 2: Negative control: $E$. coli ATCC 25922; 3 and $3 *$ : Tests with discrepant results for the same $P$. stuartii strain (negative MHT and positive mCIM test result)

Isolation of multiple CP-CRE strains from individual patients admitted to the intensive care unit (ICU)

In two patients from the same room, one of them previously hospitalized in another hospital from other Romanian geographical region, we isolated $K$. pneumoniae KPC and $P$. stuartii NDM from bronchial secretions. In one patient we detected $E$. coli OXA-48-like+ESBL and $K$. pneumoniae OXA-48-like+ESBL simultaneously from wound. In the other patient with congenital neuromusculare disease, we isolated successively $K$. pneumoniae OXA-48-like+ESBL from urine, wound and bronchial secretions.

\section{Antimicrobial susceptibility profiles by car- bapenemase gene}

Among our CP-CRE strains 100\% were resistant to ertapenem, $94.73 \%$ to imipenem and $89.47 \%$ to meropenem (Table 1 ). Only 4 OXA48-like+ESBL isolates displayed a meropenem $\mathrm{MIC} \leq 8 \mathrm{mg} / \mathrm{L}$ and the rest of CP-CRE strains exhibited high level resistance (MIC $\geq 16 \mathrm{mg} / \mathrm{L}$ ) (Figure 2).

Our investigation revealed that all 9 KPC-positive strains were resistant to aminopenicillins, to third and fourth-generation cephalo- sporins, to fluoroquinolones, aztreonam, sulfonamides, and gentamicin displayed in vitro activity against 7 of these strains.

NDM-positive strains presented resistance to almost all classes of antibiotics and fewer strains were resistant to amikacin, gentamicin, aztreonam, nitrofurantoin and colistin.

The OXA-48-like+ESBL strains were resistant to third and fourth-generation cephalosporines, piperacillin-tazobactam, aztreonam and exhibited susceptibility to amikacin, tigecycline and colistin.

Three (1 KPC and 2 OXA-48-like+ESBL) out of $9 \mathrm{~K}$. pneumoniae strains tested against colistin were found resistant (MIC $\geq 16 \mathrm{mg} / \mathrm{L}$ ). Of $9 \mathrm{~K}$. pneumoniae isolates tested against tigecycline we noted $1 \mathrm{KPC}$ strain resistant (MIC $\geq 8$ $\mathrm{mg} / \mathrm{L})$.

\section{Discussions}

In recent years, we have witnessed the emergence and worldwide dissemination of the CPCRE strains $(1,2,5,6,15)$. Both clonal spread and plasmid-mediated transmission contribute to the ongoing rise in incidence of these bacteria (1, $2,15)$. The emergence and spread of CP-CRE 
Table 1. Antimicrobial susceptibilities of CP-CRE strains by carbapenemase-encoding gene detected

\begin{tabular}{|c|c|c|c|}
\hline \multirow[b]{2}{*}{ Antimicrobial agent } & \multicolumn{3}{|c|}{$\begin{array}{c}\text { No. of susceptible and intermediate susceptible isolates, by } \\
\text { carbapenemase gene detected }\end{array}$} \\
\hline & $b l a_{\mathrm{KPC}}(\mathrm{n}=9)$ & $b l a_{\mathrm{NDM}}(\mathrm{n}=5)$ & $\begin{array}{c}\boldsymbol{b l a}_{\text {OXA-48-like }}+ \\
\text { ESBL } \\
(\mathbf{n}=5)\end{array}$ \\
\hline \multicolumn{4}{|l|}{ Carbapenems } \\
\hline Meropenem & 0 & 0 & 2 \\
\hline Imipenem & 0 & 0 & 1 \\
\hline Ertapenem & 0 & 0 & 0 \\
\hline \multicolumn{4}{|l|}{ Cephems } \\
\hline Cefuroxime & 0 & 0 & 0 \\
\hline Ceftazidime & 0 & 0 & 0 \\
\hline Cefotaxime & 0 & 0 & 0 \\
\hline Cefepime & 0 & 0 & 0 \\
\hline \multicolumn{4}{|l|}{$\beta$-Lactamase inhibitor combinations } \\
\hline \multicolumn{4}{|l|}{ Amoxicillin+clavulanic acid } \\
\hline Piperacillin+tazobactam & 0 & 0 & 0 \\
\hline & 0 & 0 & 0 \\
\hline \multicolumn{4}{|l|}{ Fluoroquinolones } \\
\hline Ciprofloxacin & 0 & 0 & 0 \\
\hline \multicolumn{4}{|l|}{ Aminoglycosides } \\
\hline Gentamicin & 7 & 1 & 1 \\
\hline Amikacin & 0 & 1 & 5 \\
\hline Tobramycin & 0 & 0 & 0 \\
\hline Trimethoprim-sulfamethoxazole & 0 & 0 & 0 \\
\hline \multicolumn{4}{|l|}{ Monobactams } \\
\hline Aztreonam & 0 & 1 out of 3 tested & 0 \\
\hline \multicolumn{4}{|l|}{ Nitrofuran } \\
\hline $\begin{array}{l}\text { Nitrofurantoin (reported only } \\
\text { for urinary isolates) }\end{array}$ & $\begin{array}{c}0 \\
(\mathrm{n}=1 \text { urinary isolate })\end{array}$ & $\begin{array}{c}1 \\
(\mathrm{n}=3 \text { urinary isolates })\end{array}$ & $\begin{array}{l}\text { Non-urinary } \\
\text { isolates }\end{array}$ \\
\hline Colistin* & 3 out of 4 strains tested & 2 & 3 \\
\hline Tigecycline* & 3 out of 4 strains tested & 1 (1 isolate untested $)$ & 5 \\
\hline
\end{tabular}

*Including natural resistance of $P$. stuartii and $S$. marcescens

were found to be related to different factors: antimicrobial use exerts ecological pressure on bacteria, poor infection control measures, cross-border transfer of patients, travel, medical tourism and international migration (6).

The most common CP-CRE species isolated in our study were K. pneumoniae. In 2013, the US Centers for Disease Control and Prevention (CDC) reported similar distribution (16). Also, the resistance situation for $K$. pneumoniae remains problematic, especially in countries in the Southern and Eastern parts of Europe (17). In 2016, the proportion of invasive carbapenem resistant $K$. pneumoniae isolates reported to the European Antimicrobial Resistance Surveillance Network EARS-Net varied from $<1 \%$ in some of the Nordic countries to $25-50 \%$ in Italy, Romania, and more than 50\% in Greece (17). Data 


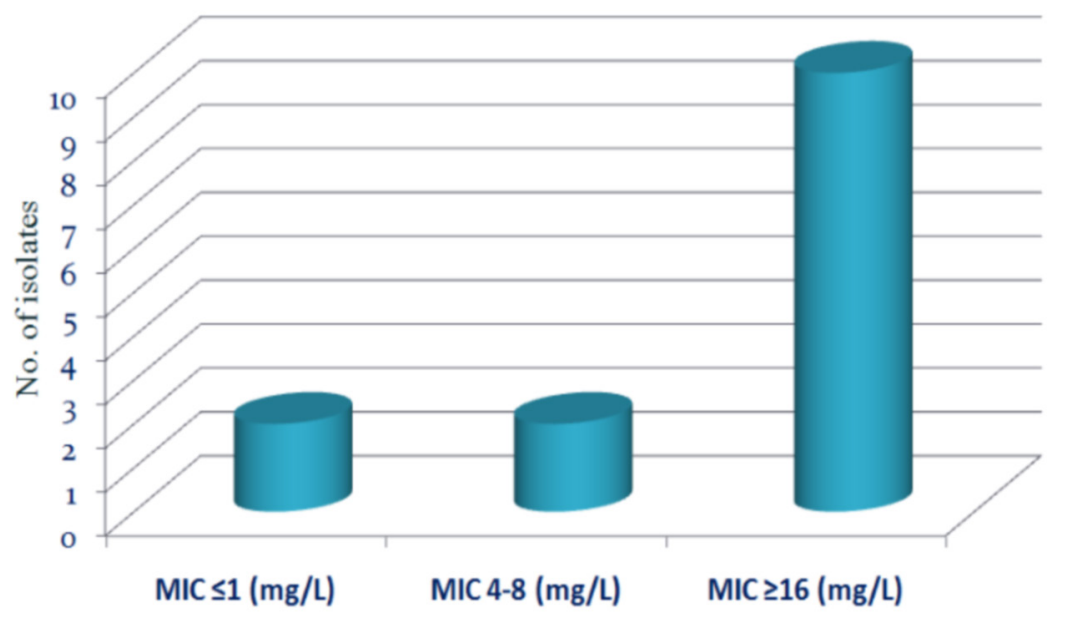

Fig. 2. Distribution of meropenem MIC for 14 CP-CRE strains (the MIC was determined in 14 strains out of the total of 19)

from EARS-Net showed a correlation between national antimicrobial consumption levels and resistance to the same antimicrobial groups in $K$. pneumoniae (6).

In our study, carbapenemases were responsible for carbapenem resistance in 19 isolates $(44.18 \%)$. The remaining $55.81 \%$ strains were most likely with reduced carbapenem susceptibility due to a dual resistance mechanisms: a cephalosporinase or ESBLs associated with porin mutations, loss of porin expression or efflux mechanism $(1,3)$.

Our study highlights that the majority of CPCRE strains harboured gene $b l a_{\mathrm{KPC}}$. The epidemiology of CRE varies by continent, country, region, and even center-to-center levels $(2,15)$. Of all carbapenemase families encountered in Enterobacteriacea, KPC carbapenemases have the most extensive global distribution (15). In the United States KPC is the predominant carbapenemase and KPC-producers are unevenly distributed among the U.S. states $(2,15)$. KPC are also widespread in South and Central America, while Europe Italy and Greece reported an endemic occurence of KPC in 2014-2015 (15).
KPC enzymes have also been found in Israel, China, Tanzania and South Africa (15).

Previous studies conducted in different Romanian geographical areas suggested that OXA-48-like and NDM were the main prevalent carbapenemases detected, while KPC enzymes were identified only sporadically (3, 9-13). In Europe NDM producers are most commonly found in Romania, Poland and Denmark, where "inter-regional spread" (epidemiological stage 4 ) is deemed to be present (18).

To our knowledge, this is the first Romanian report, where KPC was the main carbapenemase detected. Notably, we remarked the first occurrence in our hospital of $P$. stuartii harbouring gene $b l a_{\mathrm{NDM}}$. K. pneumoniae and E. coli isolates are the most commonly mentioned NDM-producers (5), nevertheless an increasing number of Enterobacteriaceae genera including C. freundii, M. morganii, E. cloacae, $K$. oxytoca have been found carrying $b l a_{\mathrm{NDM}}$ as a consequence to its particularly highly mobile encoding conjugative plasmid which facilitate horizontal interand intraspecies transfer between bacteria rather than clonal spread (19). 
Phenotypic detection of CP-CRE is complicated by the fact that the level of carbapenem resistance induced by the expression of carbapenemases is heterogeneous $(5,6)$ and there is no single phenotypic or genotypic test being suitable for all situations $(2,20)$. Detection of carbapenemases in isolates based only on MIC values has low sensitivity and specificity (20).

MHT is simple and inexpensive to perform and has already demonstrated great ability to detect KPC producers, as well as VIM, IMP, and OXA-48-like enzymes (2). In our study, MHT was positive for all KPC and OXA-48-like isolates tested using both meropenem and ertapenem disks.

Also, our results revealed 2 strains of $P$. stuartii, 1 strain of $S$. marcescens and 1 isolate of $K$. pneumoniae harbouring gene $b l a_{\mathrm{NDM}}$, which were negative for the MHT with both meropenem and ertapenem disks. Similar reports recognized the MHT unsatisfying performance in the detection of NDM-carriers, with sensitivity below 50\% $(2,8,14,21,22)$. Recent studies demonstrated that NDM is a lipoprotein anchored to the outer membrane in Gram-negative bacteria, unlike all other known carbapenemases (21). Pasteran et al. demonstrated that false negative results with MHT appear in NDM variants with this cellular localization and proposed a simple improvement of MHT, called the Triton Hodge Test (THT), by addition of a nonionic surfactant during the test (21). Also, Sun et al. reported an improvement in detection sensitivity of NDM-producers from $65.2 \%$ with MHT to $91.3 \%$ with the THT (22).

A newer phenotypic method for detection of carbapenemase activity is the mCIM (14). Several studies demonstrated high sensitivity of mCIM for CP-CRE screening $(2,4,8)$. The $\mathrm{mCIM}$ is an affordable assay using familiar materials and methods and the test can be interpreted objectively (8). Our findings with mCIM and the combination disks test were in accordance with multiplex PCR results. Molecular techniques re- main the gold standard for precise identification of carbapenemase genes (23).

The CLSI and the European Committee on Antimicrobial Susceptibility Testing EUCAST have recommended reporting of susceptibility in accordance with the antimicrobial susceptibility test result $(5,8,14)$. Detection of carbapenemase activity is undeniably important for epidemiological and infection control purposes and many would argue that it would be beneficial when making treatment decisions $(8,20)$.

In our findings, consistent with previous reports $(3,5,6,12,20,24)$, only few antibiotics remained effective against some of our CP-CRE strains: gentamicin, colistin, tigecycline. We observed 7 KPC-producing strains susceptible or intermediate susceptible to gentamicin and simultaneously resistant to amikacin. Ko et al. showed that gentamicin was not an adequate treatment option for amikacin-resistant $K$. pneumoniae, when the antibiotic susceptibility tests were performed by Vitek 2. The same authors experienced gentamicin treatment failure in $K$. pneumoniae bacteraemia that was susceptible to gentamicin despite amikacin resistance, as determined by Vitek 2. The false gentamicin susceptibility was associated with the armA 16S rRNA methylase gene (25).

Current clinical evidence for treatment guidance is limited and based on retrospective observational studies and case reports $(5,20)$. Combination regimens including colistin, carbapenems, tigecycline, aminoglycosides and fosfomycin have been used $(5,20)$. Many CP-CRE isolates may present with discrete elevations of MICs for carbapenems and these drugs were used as potential treatment options in high-dose regimens $(5,20)$. Unfortunately, in most of our tested strains high level resistance was noted to meropenem (MIC $\geq 16 \mathrm{mg} / \mathrm{L}$ ), which compromises the possibility of synergistic action of using meropenem in an antibiotic combination scheme 
(3). Daikos et al. found that patients treated for carbapenemase-producing $K$. pneumoniae bacteraemia with a carbapenem $\mathrm{MIC} \leq 8 \mathrm{mg} / \mathrm{L}$ in combination with other active agents had a lower mortality than those with a MIC $>8 \mathrm{mg} / \mathrm{L}(19.3 \%$ versus $35.5 \%$ ) (24).

With the emergence of resistance to colistin and tigecycline, some of the few remaining treatment alternatives for patients infected with CP-CRE, represent a major concern $(18,26)$. We noted 3 strains of $K$. pneumoniae resistant to colistin recovered from bronchial secretion and wounds. Also, we observed 1 KPC-producing strain from bloodstream infection resistant to tigecycline $(\mathrm{CMI} \geq 8 \mathrm{mg} / \mathrm{L})$. Polymyxin resistance, which is usually chromosomally mediated leading to modification of lipid A, was recently found to occur through acquisition of a plasmid-mediated gene, $m c r 1$ (26). In EARSNet report for 2016, Greece and Romania reported a combined resistance to carbapenems and colistin between 10-25\% (17). However, as routine colistin susceptibility testing has several methodological issues, the extent of the problem is difficult to assess based on EARS-Net data (17). Recently, Chiu et al. demonstrated that tigecycline resistance may occur without previous exposure to tigecycline and resistance mechanisms to tigecycline are not limited to increased expression of efflux pump genes such as acrB and/or oqxB (27).

Avoidance of inappropriate antimicrobial use and comprehensive infection prevention and control strategies targeting all healthcare sectors are the key measures of effective interventions aiming to prevent selection and transmission of bacteria resistant to antimicrobial agents (17).

\section{Limitations of the study}

Epidemiological and clinical data were not collected. Detection of cephalosporinase overex- pression, porin mutations and efflux mechanism were not confirmed by laboratory analysis.

\section{Conclusions}

We have described the emergence of CPCRE strains in a non-teaching Romanian hospital located in the North of the country. Although these have been reported from many large Romanian university hospitals since 2012, their presence in non-teaching hospitals is not very well known, but their spread is to be expected via patient transfers between hospitals. Awareness of this phenomenon, rapid and reliable detection of CP-CRE is critical for preventing their further spread in these settings.

\section{Acknowledgments}

Results of this study were presented in part at the $10^{\text {th }}$ National Conference of Microbiology and Epidemiology, 2-4 ${ }^{\text {th }}$ November 2017, Bucharest, Romania. We thank Dr. Szász Izabella, Dr. Vas Krisztina, Dr. Truţa Andreea, Dr. Pocol Oana, Dr. Romanschi Andrei for their technical assistance.

\section{Authors' Disclosures of Potential Conflicts of Interest}

No potential conflicts of interest relevant to this article were reported. This study was not funded.

\section{Author contributions}

Conceived and designed the experiments: FA, SE. Performed the experiments: FA, SE. Analyzed the data: FA, SE. Wrote the paper: FA. Critically revised the paper: SE, BDV.

\section{References}

1. Nordmann P, Dortet L, Poirel L. Carbapenem resistance in Enterobacteriaceae: here is the 
storm! Trends in Mol Med 2012;18(5):263-72. DOI: 10.1016/j.molmed.2012.03.003

2. Lutgring JD, Limbago BM. The problem of carbapenemase-producing-carbapenem-resistant Enterobacteriaceae detection. J Clin Microbiol 2016;54:529-534. DOI: 10.1128/JCM.02771-15

3. Popescu GA, Codiţă I, Szekely E, Şerban R, Ruja G, Tălăpan D. Ghid privind Enterobacteriaceae producătoare de carbapenemaze: diagnosticul, prevenirea transmiterii interumane şi tratamentul infecţiilor produse. Centrul Naţional de Supraveghere şi Control a Bolilor Transmisibile 2015. Available from: http:// www.spcin.ro/ghiduri-537i-metodologii.html.

4. Yamanda K, Kashiwa M, Arai K, Nagano N, Saito R. Evaluation of the modified carbapenem inactivation method and sodium mercaptoacetate-combination method for the detection of metallo- $\beta$-lactamase production by carbapenemase-producing Enterobacteriaceae. J Microbiol Meth 2017;132:112-115. http://dx. doi.org/10.1016/j.mimet.2016.11.013.

5. Tangdén T, Giske CG. Global dissemination of extensively drug-resistant carbapenemase-producing Enterobacteriaceae: clinical perspectives on detection, treatment and infection control. J Intern Med 2015;277:501-512. DOI: 10.1111/joim.12342

6. European Centre for Disease Prevention and Control. Rapid risk assessment: carbapenem-resistant Enterobacteriaceae - 8th April 2016. Stockholm: ECDC; 2016. Available from: https://ecdc.europa.eu.

7. World Health Organization. Global priority list of antibiotic-resist $\neg$ ant bacteria to guide research, discovery, and development of new antibiotics. Geneva: WHO; 2017. Available from: http://www.who.int/medicines/ publications/WHO-PPL-Short_Summary_25Feb-ET_ NM_WHO.pdf

8. Butler-Wu SM, Abbott AN. Is this the carbapenemase test we've been waiting for? A multi-center evaluation of the Modified Carbapenem Inactivation Method (mCIM). J Clin Microbiol 2017;55:2321-2333. DOI: 10.1128/JCM.00538-17

9. SzékelyE,DamjanovaI,JánváriL, VasKE,MolnárS,Bilca DV, et al. First description of blaNDM-1, blaOXA-48, blaOXA-181 producing Enterobacteriaceae strains in Romania. Int J of Med Microbiol 2013;303(8):697-700. DOI: 10.1016/j.ijmm.2013.10.001

10. Dortet L, Flonta M, Boudehen YM, Creton E, Bernabeub S, Vogel A, et al. Dissemination of car- bapenemase-producing Enterobacteriaceae and Pseudomonas aeruginosa in Romania. Antimicrob Agents Chemother 2015;59(11):7100-7103. DOI: 10.1128/AAC.01512-15

11. Lixandru BE, Cotar AI, Straut M, Usein CR, Cristea D, Ciontea $\mathrm{S}$, et al. Carbapenemase-producing Klebsiella pneumoniae in Romania: A SixMonth Survey. PLoS ONE 2015;10(11): e0143214. DOI: 10.1371/journal.pone.0143214

12. Rafila A, Talapan D, Dorobăţ OM, Popescu GA, Piţigoi D, Florea D, et al. Emergence of carbapenemase-producing Enterobacteriaceae, a public health threat: a Romanian infectious disease hospital based study. Revista Română de Medicină de Laborator 2015;23(3):295-301. DOI: 10.1515/rrlm-2015-0024

13. Popescu C, Popescu GA, Dorobăț O, Rafila A, Tănase D, Mikula C, et al. OXA-48-carbapenemase-producing Klebsiella pneumoniae infections - the first cases diagnosed in Romanian National Institute of Infectious Diseases. Revista Română de Medicină de Laborator 2017;25(1):55-61. DOI: $10.1515 / \mathrm{rrlm}-2017-0006$

14. Performance Standards for antimicrobial susceptibility testing, $27^{\text {th }}$ informational supplement. CLSI document M100-S27. Clinical and Laboratory Standards Institute, 2017.

15. Duin vD, Doi Y. The global epidemiology of carbapenemase-producing Enterobacteriaceae. Virulence 2017;8(4):460-469. DOI: 10.1080/21505594.2016.1222343

16. Centers for Disease Control and Prevention. Vital signs: carbapenem-resistant Enterobacteriaceae. MMWR Morbid. Mortal. Wkly. Rep. 2013;62(09):165-170.

17. European Centre for Disease Prevention and Control. Surveillance of antimicrobial resistance in Europe 2016. Annual report of the European Antimicrobial Resistance Surveillance Network (EARS-Net), November 2017. Available from: https://ecdc.europa.eu.

18. Albiger B, Glasner C, Struelens MJ, Grundmann $\mathrm{H}$, Monnet DL, the European Survey of Carbapenemase-Producing Enterobacteriaceae (EuSCAPE) working group. Carbapenemase-producing Enterobacteriaceae in Europe: assessment by national experts from 38 countries, May 2015. Euro Surveill 2015;20(45):piiD30062;PMID:26675038. DOI: 10.2807/15607917. ES.2015.20.45.30062

19. Wang X, Chen G, Wu X, Wang L, Cai J, Chan 
EW, et al. Increased prevalence of carbapenem resistant Enterobacteriaceae in hospital setting due to cross-species transmission of the blaNDM-1 element and clonal spread of progenitor resistant strains. Front. Microbiol. 2015;6:595. DOI: $10.3389 /$ fmicb.2015.00595

20. Bano JR, Cisneros JM, Cobos-Trigueros N, Fresco G, Navarro-San Francisco C, Gudiol C, et al. Diagnosis and antimicrobial treatment of invasive infections due to multidrug-resistant Enterobacteriaceae. Guidelines of the Spanish Society of Infectious Diseases and Clinical Microbiology. Enferm Infecc Microbiol Clin 2015;33(5):337.e1-337.e21. DOI: 10.1016/j. eimc2014.11.009

21. Pasteran F, Gonzalez LJ, Albornoz E, Bahr G, Vila AJ, Corso A. Triton Hodge Test: Improved protocol for modified Hodge test for enhanced detection of NDM and other carbapenemase producers. J Clin Microbiol 2016;54(3):640-649. DOI: 10.1128/JCM.01298-15

22. Sun K, Xu X, Yan Y, Zhang L. Evaluation of six phenotypic methods for the detection of carbapenemases in Gram-negative bacteria with characterized resistance mechanisms. Ann Lab Med 2017;37:305-312. DOI: 10.3343/alm.2017.37.4.305

23. Nordmann P, Poirel L. Strategies for identification of carbapenemase-producing Enterobacteriaceae. J Antimicrob Chemother 2013;68:487-489.
DOI: $10.1093 / \mathrm{jac} / \mathrm{dks} 426$

24. Daikos GL, Tsaousi S, Tzouvelekis LS, Anyfantis I, Psichogiou M, Argyropoulou A, et al. Carbapenemase-producing Klebsiella pneumoniae bloodstream infections: lowering mortality by antibiotic combination schemes and the role of carbapenems. Antimicrob Agents Chemother 2014;58(4):2322-2328. DOI: 10.1128/AAC.02166-13

25. Ko JH, Baek JY, Peck KR, Cho SY, Ha YE, Kim $\mathrm{SH}$, et al. Discrepant susceptibility to gentamicin despite amikacin resistance in Klebsiella pneumoniae by VITEK 2 represents false susceptibility associated with the armA 16S rRNA methylase gene. J Med Microbiol 2017;66(10):1448-1450. DOI: 10.1099/jmm.0.000583

26. Liu YY, Wang Y, Walsh TR, Yi LX, Zhang R, Spencer J, et al. Emergence of plasmid-mediated colistin resistance mechanism MCR-1 in animals and human beings in China: a microbiological and molecular biological study. Lancet Infect Dis 2015;16:161-8; PMID:26603172. DOI: 10.1016/S1473-3099(15)00424-7

27. Chiu SK, Chan MC, Huang LY, Lin YT, Lin JC, $\mathrm{Lu} \mathrm{PL}$, et al. Tigecycline resistance among carbapenem-resistant Klebsiella pneumoniae: Clinical characteristics and expression levels of efflux pump genes. PloS ONE 2017;12(4):e0175140. DOI: 10.1371 /journal.pone.0175140 